Archives

10 | 1993

Le Centre de recherches historiques de 1949 à 1975

\title{
Le triomphe d'un modèle : 1965-1973
}

\section{OpenEdition}

Journals

\section{Édition électronique}

URL : http://journals.openedition.org/ccrh/2790

DOI : $10.4000 /$ ccrh. 2790

ISSN : 1760-7906

Éditeur

Centre de recherches historiques - EHESS

\section{Édition imprimée}

Date de publication : 15 avril 1993

ISSN : 0990-9141

Référence électronique

"Le triomphe d'un modèle : 1965-1973 », Les Cahiers du Centre de Recherches Historiques [En ligne],

10 | 1993, mis en ligne le 16 mars 2009, consulté le 19 avril 2019. URL : http://

journals.openedition.org/ccrh/2790; DOI : 10.4000/ccrh.2790

Ce document a été généré automatiquement le 19 avril 2019

Article L.111-1 du Code de la propriété intellectuelle. 


\section{Le triomphe d'un modèle : 1965-1973}

1 Il est commode de choisir l'année 1965 comme date symbolique marquant la fin de cette phase de transition: c'est l'année de la consécration internationale des activités de recherche du CRH aux deux congrès internationaux de Munich et de Vienne. C'est aussi l'année d'un changement significatif à la tête du CRH: R. Romano, l'un des fidèles collaborateurs de F. Braudel et qui joua les premiers rôles aux débuts du CRH, démissionne du poste de secrétaire où $\mathrm{E}$. Le Roy Ladurie lui succède, symbole de la fin de la phase «méditerranéenne» ou «internationale» du Centre. Les collaborateurs espagnols, italiens et latino-américains se firent désormais plus rares, l'ancien réseau de collaborateurs étrangers étroitement liés à la personne et aux recherches de F. Braudel fut successivement remplacé par un réseau essentiellement français d'agrégés et de normaliens passés devant les jurys d'agrégation présidés par Braudel de 1950 à 1956 et qui ont commencé leurs recherches en disciples formels ou réels d'E. Labrousse. Les nominations de F. Furet, E. Le Roy Ladurie, J. Le Goff et P. Jeannin comme directeurs d'études témoignent de l'arrivée de toute une génération nouvelle d'historiens français marqués par l'influence intellectuelle et le poids universitaire (en termes de nominations ou de crédits) de Labrousse et de Braudel pendant les années 1950. Si l'on fait abstraction de son enseignement à la VI Section, la présence d'E. Labrousse au CRH même remonte à la fin des années 1950. L'enquête sur les sources de l'histoire des structures sociales des $\mathrm{XVIII}^{\mathrm{e}}$ et XIX ${ }^{\mathrm{e}}$ siècles, lancée par la Commission internationale d'histoire des mouvements sociaux et des structures sociales en 1956-7, était financée par le CRH. Elle ouvrait la voie au dépouillement, animé par A. Daumard, des archives de l'enregistrement ${ }^{1}$. Autres précurseurs: les équipes déjà mentionnées de $\mathrm{B}$. Gille et de J. Bouvier travaillant sur l'histoire du profit au XIX ${ }^{e}$ siècle et sur l'histoire des entreprises.

2 Sous la direction d'E. Le Roy Ladurie à partir de 1965, puis de F. Furet à partir de 1970, des recherches inspirées par les propositions de Labrousse pour une histoire économique et sociale des origines de la France bourgeoise, trouvaient une place centrale dans le programme du CRH. Ce qui caractérise ces projets issus du fonds labroussien, c'est leur longévité : l'enquête sur les sources de l'histoire des structures sociales a été prolongée par celle sur les fortunes privées des français au XIX ${ }^{\mathrm{e}}$ siècle qui a suscité toute une série de 
recherches sur la sociologie urbaine de Paris au XIX ${ }^{e}$ siècle, lesquelles en ont enfanté d'autres sur la croissance urbaine au xix ${ }^{e}$ siècle ou sur les classes dirigeantes dans la France contemporaine. Les enquêtes sur les grands notables et sur la mobilité sociale aux $\mathrm{XVII}^{\mathrm{e}}$ et XVIII ${ }^{\mathrm{e}}$ siècles rejoignent celles d'A. Tudesq entre 1957 et 1964 sur les grands notables de la monarchie censitaire.

La longévité du programme labroussien était liée à la place du réseau de ses disciples dans le champ universitaire. Le contrôle qu'E. Labrousse a exercé pendant ces années sur le recrutement universitaire en tant que directeur et membre des jurys de thèses parisiens et sur les ressources de recherche en tant que membre influent de la section d'histoire moderne et contemporaine du comité national du $\mathrm{CNRS}^{2}$ lui a permis de veiller à la carrière de bon nombre de ses élèves. Le fait que ce réseau était surtout national constitue sa différence par rapport au monde braudelien largement cosmopolite des années 1950 et 1960 : tout à la fois les terrains de recherche, les traditions intellectuelles (Simiand et un certain style d'histoire sociale dont J. Jaurès restait le modèle) et les positions politiques (une gauche socialiste et communiste) du monde labroussien étaient axés sur la France ${ }^{3}$. Au moment où la $\mathrm{VI}^{\mathrm{e}}$ Section commença à offrir des postes et des perspectives de carrière universitaire, ce réseau labroussien profita largement de sa rente de situation qui lui offrait un double choix : les portes universitaires et celles de la $\mathrm{VI}^{\mathrm{e}}$ Section lui étaient largement ouvertes.

4 L'arrivée de la deuxième génération annaliste aux postes de direction du CRH sonnait l'heure d'une réorganisation des programmes de recherche et d'une redéfinition des ambitions intellectuelles. À partir de 1966, « Histoire quantitative » et « Anthropologie historique » devinrent les deux étiquettes qui devaient regrouper les différents champs de recherche et coordonner, au moins métaphoriquement, les initiatives de la nouvelle direction. La demande d'association au CNRS en 1966 fut l'occasion de recentrer les activités du CRH autour d'un nouveau projet principal baptisé « Anthropologie culturelle de la France préindustrielle» (sous la responsabilité de Le Roy Ladurie et Furet), regroupant sous deux rubriques - «Écologie historique » et « Niveaux de culture » - toute une gamme d'enquêtes d'histoire moderne et médiévale sur les espaces culturels, les données biologiques, la culture matérielle (habitat traditionnel, alimentation), les structures familiales, l'inconscient collectif, l'histoire du livre et des intellectuels des lumières. 


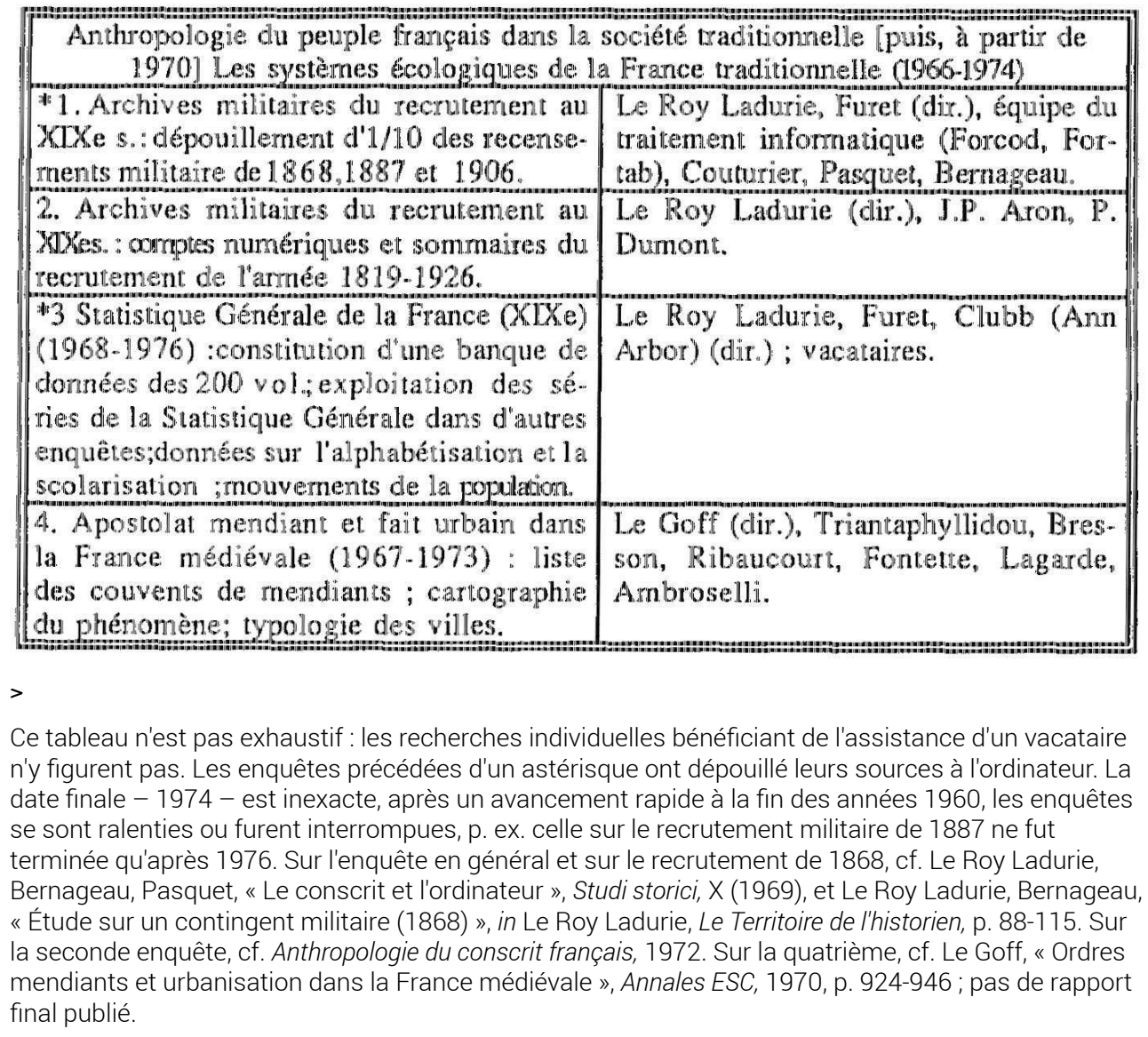

Ce projet d'une description quantitative détaillée - dans les dimensions de leur évolution temporelle globale et de leur distribution géographique - des différents étages de l'aire culturelle de la France traditionnelle - du biologique et de la culture matérielle aux phénomènes religieux et intellectuels - permettait de réintégrer toute une série de recherches en cours et de les poursuivre sous de nouvelles étiquettes. Même dans sa première version, celle de 1965-66, le nouveau programme du CRH cherchait à intégrer les acquis de la phase précédente. Les enquêtes qui avaient eu un retentissement international, comme celle des archéologues médiévistes et celle sur le livre et la société se poursuivaient. Autre signe de continuité : le CRH continua de s'engager dans des projets d'enquêtes nationales ou internationales en coordonnant les équipes et en préparant les congrès. Ce fut le cas de l'enquête sur la production agricole selon les dîmes, d'abord national puis repris en 1973 au niveau international, et celui des enquêtes sur la consommation ou sur les fortunes françaises au XIX ${ }^{\mathrm{e}}$ siècle.

6 Un tel programme impliquait d'opter pour des enquêtes sur de grandes masses de données exigeant des traitements statistiques ultérieurs et demandant l'aide technique d'ordinateurs : «Grâce à la révolution introduite par l'ordinateur dans le maniement de l'information, il [le CRH] développe aujourd'hui son activité vers le dépouillement de plus en plus systématique des données quantitatives de l'histoire, et la mise eu point des procédures d'exploitation les plus sures et les plus rapides ${ }^{4}$. " L'aspect technique de la recherche gagnait sous l'étiquette " histoire quantitative » une valeur autonome comme symbole de la nouvelle scientificité de la recherche historique. Bien que les directeurs des 
programmes de recherche eussent manifesté de bonne heure leur prudence épistémologique ${ }^{5}$, il n'empêchait que le texte des enquêtes, le choix des sujets et les déclarations programmatiques fussent imprégnés d'un scientisme activiste, avec sa foi dans les promesses des nouvelles techniques de traitement des sources et de fabrication des résultats : l'entrée de la recherche historique dans l'ère de l'ordinateur exigeait, selon le discours unificateur de la nouvelle direction du CRH, à la fois la création de nouveaux moyens de travail, à savoir les banques de données et un nouveau système d'archivage électronique - qui devait être réalisé grâce à l'aide technique et financière de l'université d'Ann Arbor et de son Survey Research Center. En liant le vieux projet d'une histoire économique et sociale à base statistique aux innovations technologiques de l'informatique, la nouvelle direction reformulait l'image d'avant-garde du CRH dans un langage à connotations scientistes et technicistes.

7 Le programme d'histoire sérielle sur ordinateur eut une existence plutôt difficile. Avec la création d'un atelier de perforation et le recrutement d'une équipe d'informaticiens autour de M. Couturier en 1966, le traitement informatique des masses de données des enquêtes sérielles commença. Jusqu'à la fin des années 1970, les systèmes Forcod et Fortab propres au CRH ont été successivement mis au point pour servir de base au traitement informatique des registres du recrutement militaire, des données sur l'armée révolutionnaire, sur les notables du Grand Empire et les cahiers de doléances. 
Tableau 10. Enquêtes collectives commencées entre 1965-1968. II

\begin{tabular}{|c|c|}
\hline \multicolumn{2}{|c|}{ Maison et bâtiment dans l'histoire économigue: $(1965-19 \%)$} \\
\hline $\begin{array}{l}\text { "Recherches sur les loyers et le revenu dans } \\
\text { l'industrie du batiment ; conurbes des loyers pa- } \\
\text { risiens du XVe au XVIIIe s, sur ordinateur. }\end{array}$ & Le Roy Ladurie (dir.), Couperie \\
\hline \multicolumn{2}{|c|}{ "Livre et société dans la France du XVDIle s. III (1965-1970) } \\
\hline $\begin{array}{l}\text { IDfpouillement des registres de commandes de } \\
\text { livres (1779-1789); analyse desérnantique his- } \\
\text { torigue des titres d'ouvrages dans les registres } \\
\text { de lladministration royale de la libratrie;le mi- } \\
\text { lieu de l'Encyclopédie et des Académies. }\end{array}$ & 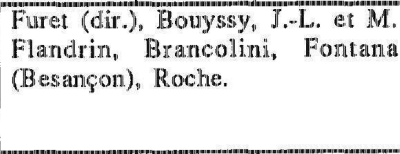 \\
\hline \multicolumn{2}{|c|}{ Enquête sur les archives de l'Academie de Médecine (1955-1969) } \\
\hline $\begin{array}{l}\text { "1. La météorologie de la France : exploita- } \\
\text { thon mécaunographique des données climatiques } \\
\text { dans les archives Vicg d'Azir. }\end{array}$ & $\begin{array}{l}\text { Lee Roy Ladurie (dir, , Desaive, Bau- } \\
\text { dry, Muller (MSH) }\end{array}$ \\
\hline 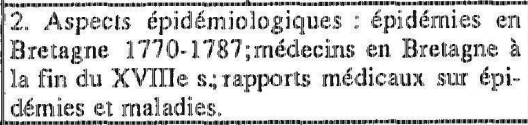 & Goulbent, Meyer (Rermes), Peter \\
\hline \multicolumn{2}{|c|}{ Les structures economiques et sociales de l'époque moderne $(1965-1970)$} \\
\hline $\begin{array}{l}\text { 1. Histoine de la production agricole darn la } \\
\text { France moderne d'après les dinnes (1966- } \\
\text { 1969): dépouillement. des archives départe- } \\
\text { mentales pour la construction d'une série de } \\
\text { dîmes régionales ; traitement statistique des } \\
\text { donmées brutes. }\end{array}$ & $\begin{array}{l}\text { Labrousse (dir.), Le Roy Ladurie, } \\
\text { Goy, Desaive, Baulant, Veyrassats- } \\
\text { Henren, Koenig, Silber fi, nombreum } \\
\text { collaborateurs extérieurs. }\end{array}$ \\
\hline $\begin{array}{l}\text { 2. Fortune privée des Français au XTXe s. } 181.5 \text { - } \\
\text { 1914 }(1964-1970) \text { : dépouillement des déclara- } \\
\text { lions de succession de Paris (1911); regroupe-- } \\
\text { ment des donúes ; répartition des niveaux de } \\
\text { fortune, composition des fortunes selon les } \\
\text { catégories socio-professionnelless, totalisa- } \\
\text { tion des montants de fortunes. }\end{array}$ & $\begin{array}{l}\text { Daumard, Labrousse (dir., vaca- } \\
\text { taires du CRH pour les recherches } \\
\text { parisiennes ; Godechot (Toulouse), } \\
\text { Dupeux (Bordeaux), LÉn (Lyon), } \\
\text { Codaccioni (Lille). }\end{array}$ \\
\hline
\end{tabular}

L'enquête « Maison et bâtiment » est commune avec le Centre d'histoire quantitative de l'Université de Caen. Bardet, Chaunu et al., Le bâtiment, enquête d'histoire économique, T. 1, 1971 (le T. 2 n'a pas été publié) ; Le Roy Ladurie, Couperie, « Le mouvement des loyers parisiens de la fin du Moyen-Âge au XVIII e s. », Annales ESC, 1970, p. 1002-23. Boussy, Brancolini et al., Livre et société dans la France du XVIII siècle, T. 2, 1970. Desaive, Goubert, Le Roy Ladurie, Meyer, Muller, Peter, Médecins, climat et épidémies à la fin du XVIII' S., 1972. L'enquête sur la production agricole d'après les dîmes fut lancée par la Commission d'histoire moderne et contemporaine du CNRS de 1963 et a été présentée au ler Congrès de l'Association française des historiens économistes à Toulouse en 1969. Goy, Le Roy Ladurie, Les F luctuations du produit de la dîme, 1972. L'enquête sur la fortune privée a démarré en 1964 comme RCP du CNRS et fut poursuivie simultanément à Paris, Lyon, Lille, Bordeaux et Toulouse. Daumard, Les F ortunes françaises au XIXe S., 1973.

8 À côté de «la ligne Couturier » se sont développés d'autres systèmes qui utilisaient des programmes et des langages plus répandus (systèmes IBM, langage Fortran) comme pour les enquêtes de démographie historique, le cadastre florentin ou les données des Archives de l'Académie de médecine. L'archivage des données informatisées a constitué un stock considérable dont l'utilisation est pourtant restée très sporadique. Le projet initial de créer un centre de documentation informatisée d'histoire économique et sociale fut très tôt abandonné. Après une première vague d'enthousiasme, "cette rencontre d'une révolution méthodologique et d'une révolution technique ${ }^{6}$ » s'est banalisée au rythme de la diffusion des innovations informatiques en contredisant toutes les prophéties. Le traitement informatique de masses toujours croissantes de données s'avérait plus lent et plus compliqué que prévu, l'organisation de la saisie sur ordinateur butait souvent sur le manque de vacataires voués à ces travaux routiniers. Le savoir informatique restait alors le monopole d'un groupe restreint autour des informaticiens programmateurs. De plus, le rendement scientifique des séries sorties des ordinateurs n'était pas toujours à la mesure des ressources engagées dans l'enquête. Mais au fond, le CRH d'après 1965 et surtout d'après 1968 n'offrait pas les structures d'accueil favorables à un projet qui risquait de le 
transformer en laboratoire de service historique orienté vers des enquêtes quantitatives très longues ou des opérations de préparation et de traitement informatique de données de base pour d'autres équipes ou centres de recherche. Son personnel avait d'autres ambitions.

9 «L'anthropologie historique " était le second concept mis en avant par la nouvelle direction du CRH. Il serait intéressant d'analyser la naissance et la circulation de ce terme programmatique qui est devenu l'un des slogans les plus réussis de "l'histoire nouvelle » des années 1970. En 1966, l'idée centrale était de transférer le concept d'«aires culturelles» utilisé par les ethnologues à la recherche "des grands traits d'une géographie du développement et du sous-développement de la France ancienne avec ses répercussions sur la France présente ${ }^{7}$ ». L'élargissement de ces recherches à une "Anthropologie du Peuple Français», envisagé au début de celles sur les archives militaires, ne s'est pas produit, le champ de l'anthropologie historique s'est tout de suite fragmenté en une série de sous-champs - histoire religieuse, démographie historique, histoire des familles, histoire de la vie matérielle - qui ont défendu leur légitimité scientifique à l'intérieur du CRH en excipant de son label qui devint un lieu commun échappant à toute définition de ses objets de recherche ou de ses méthodes mais qui, en contrepartie, a pu donner une raison d'être symbolique suffisante à toute une gamme de recherches d'histoire sociale et socioculturelle (pour reprendre les termes de D. Roche) ${ }^{8}$ en les affranchissant de leurs rapports obligés avec les analyses des systèmes économiques ou politiques?.

10 L'innovation du discours programmatique du CRH donnait au niveau historiographique une première réponse aux changements dans les sciences humaines des années 1960. F. Braudel avait préparé les historiens de la $\mathrm{VI}^{\mathrm{e}}$ Section à un dialogue avec les autres sciences sociales en pleine expansion. Mais les échanges avec la sociologie restaient limités à quelques cas plutôt rares (bien que toute une série de nominations de directeurs d'études au début des années 1960 montrent l'existence d'une mode sociologisante); l'aggiornamento des disciplines littéraires sous l'impulsion structuraliste trouvait un large écho tandis que la confrontation avec une ethnologie qui avait gagné une légitimité culturelle nouvelle imposait la lecture des nouveaux maîtres à penser. Mais il est intéressant de voir que la confrontation avec les autres sciences sociales restait livresque au CRH. Avec "l'histoire nouvelle», un nouveau code de citations et de lectures s'est établi, donnant une place privilégiée aux textes anthropologiques, une nouvelle rhétorique historiographique s'est instaurée, exigeant des références hors de la discipline, mais la pratique de recherche et d'enseignement au CRH restait largement exempte du nouveau code d'interdisciplinarité promu dans les textes programmatiques. À côté du séminaire de recherche de F. Furet sur la sémantique historique de 1966-1968 et de l'enquête sur la sémantique de la correspondance de Rousseau dirigée par F. Furet et D. Roche auxquels participaient des linguistes, on ne trouve guère que l'enquête d'un géographe (M. Roncayolo) et d'un historien (L. Bergeron) sur les mécanismes de la croissance urbaine à Paris au XIX ${ }^{\mathrm{e}}$ siècle. Il faut attendre 1974 pour voir un séminaire et une enquête largement interdisciplinaires - sur l'ethno-histoire des baronnies - auxquels participaient ethnologues, linguistes, démographes, médecins et historiens.

11 La fin des années 1960 confrontait le groupe dirigeant du CRH de plus en plus avec le problème de savoir comment gérer le succès social et intellectuel de l'institution. Le poids toujours croissant des choix antérieurs, à la fois en termes de personnel et de profil intellectuel, créait une logique sociale de «la gestion responsable » qui - au niveau du 
discours - cherchait à souligner la spécificité de l'héritage intellectuel ${ }^{10}$ grandissant son prestige intellectuel par une collaboration toujours plus étroite avec les Annales ESC ${ }^{11}$ et à intégrer un personnel croissant dans des programmes de recherche plus continus. Notons au passage que dans cette situation, "la rationalité opportuniste ${ }^{12}$ " - l'offre d'un instrument nouveau pour traiter les documents d'archives des programmes antérieurs et de nouvelles sources d'archives inconnues (comme celles de l'Académie royale de Médecine) ou oubliées (comme la statistique générale de la France) - gagnait de l'importance, et cela explique d'ailleurs en grande partie le succès social d'un programme qui pour au moins 10 ans absorba une grande partie des capacités de recherche du CRH.

Le prestige intellectuel du CRH a sans aucun doute contribué à faire monter le nombre de directeurs d'études prêts à s'engager dans des enquêtes collectives de 7 en 1965 à 18 en 1971. Comme le nombre des autres collaborateurs n'augmentait pas au même rythme, cet élargissement devait faire renaître les tendances centrifuges: les listes des enquêtes s'allongèrent d'un rapport d'activités à l'autre au profit d'équipes plus petites, en même temps que les enquêtes tendaient à s'étaler sur plus de 4 ou 5 ans. De 15 enquêtes en 1969, on passe à 34 en 1974-1976 pour finir à 51 en 1978-1980. Après une phase de centralisation dont l'année 1971 représente le point culminant avec $40 \%$ des collaborateurs permanents engagés dans trois enquêtes, la tendance allait vers des groupes de recherche plus modestes de 3 à 5 chercheurs permanents. En 1974, il ne restait qu'une seule enquête absorbant 10 chercheurs, les 57 autres collaborateurs participaient à 16 enquêtes regroupant en moyenne 3,56 chercheurs ${ }^{13}$. Le triomphe du modèle de l'enquête collective commença à produire des effets d'affichage en incitant un nombre croissant de chercheurs de transformer des recherches individuelles à dimension modeste en enquête collective évitant une marginalisation à l'intérieur du monde historique de la $\mathrm{VI}^{\mathrm{e}}$ Section et profitant du crédit symbolique et financier accordé à cette forme de la recherche historique.

La dilatation des terrains de recherches imposait de plus en plus à la direction l'intégration sémantique des enquêtes en cours et la gestion d'un personnel de chercheurs permanents plus nombreux, plus âgé et plus hétérogène. Les rapports d'activités toujours plus longs du Centre entre 1968 et 1974 suggèrent d'abord l'image bien répandue de l'émiettement de la recherche historique en son sein. De fait, le recrutement de nouveaux directeurs d'études non-cumulants permettait d'ouvrir de nouveaux chantiers : sur l'histoire des $\mathrm{XVI}^{\mathrm{e}}$ et $\mathrm{XVII}^{\mathrm{e}}$ siècles (Richet), sur les origines de la France bourgeoise (Bergeron), sur l'histoire de l'enseignement et de l'alphabétisation (Ozouf). Mais il y intervient un autre effet résultant des ruptures des équilibres pré-1968 dans le monde universitaire : toute une série d'enquêtes nouvelles ne sont plus dirigées par des directeurs d'études mais par des maîtres-assistants ou des chefs de travaux qui, travaillant déjà depuis des années sur des terrains spécialisés, profitaient de la remise en cause des hiérarchies universitaires après la crise de 1968 pour gérer avec plus d'autonomie leurs propres enquêtes. Les groupes de recherche de J.-P. Goubert, J.P. Peter, J.-P. Aron, Ch. lapisch, M. Baulant, M. Keul, J.-J. Hémardinquer et W.L. Randles en témoignent.

Des groupes plus au moins stables s'établirent sans participer, ou peu, aux autres initiatives de recherche du CRH et poursuivirent leurs propres projets sur plus d'une décennie. 


\begin{tabular}{|c|c|}
\hline  & 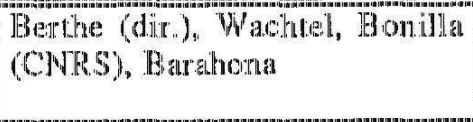 \\
\hline 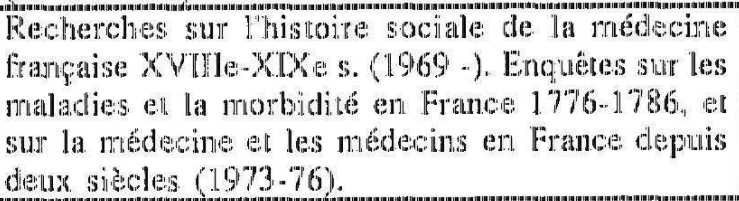 & 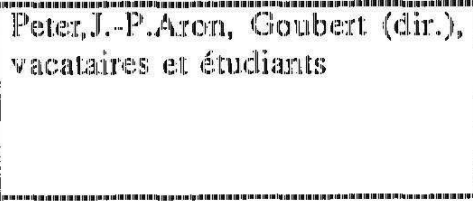 \\
\hline 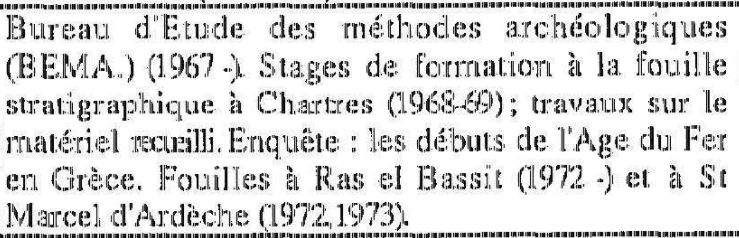 &  \\
\hline 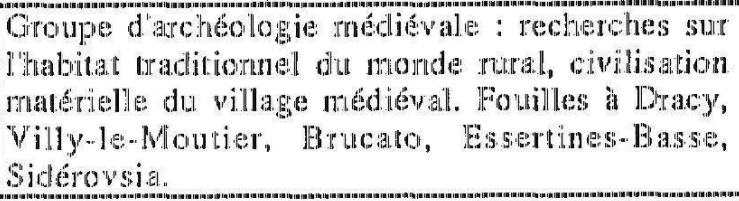 & 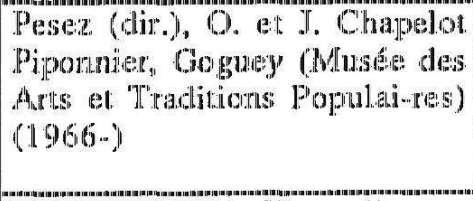 \\
\hline $\begin{array}{l}\text { Groupe de rechenches en wue de la publication d'un } \\
\text { atlas historicue mordiall des animatur domestiques } \\
\text { (1959-1977? }\end{array}$ & $\begin{array}{l}\text { Bjertin, Kenl, Hermarding,|luer, } \\
\text { R. andles. }\end{array}$ \\
\hline
\end{tabular}

Du groupe d'archéologie médiévale, cf. Pesez et al., Archéologie du village déserté, Cahiers des Annales, 2 vol., 1970

Parmi ces groupes de recherche stables, il y a d'abord les archéologues arrivés au CRH au début des années 1960 et engagés dans des recherches sur la civilisation matérielle. La renaissance d'une archéologie modernisée s'est poursuivie au sein du CRH, et les groupes archéologiques ont élargi, dans la seconde moitié des années 1970, leur champ de recherche grâce à la participation des byzantinistes. Ces groupes comme les autres avaient tendance à perpétuer leurs programmes primitifs et leur stabilité résultait largement d'un savoir technique ou thématique spécialisé qu'ils avaient accumulé au fil des années. Dans tous ces cas, les recherches restaient profondément marquées par le choix initial de la base de documentation: celles dans les archives de l'Académie de Médecine, sur les Relaciones géographicas ou sur les pèlerinages rappellent la permanence d'une recherche fortement individualisée où chercheurs et sources semblent se fondre dans un programme de travail à vie.

Mais à côté de ces groupes stables et largement autonomes, d'autres moins fixes et moins durables se sont formés et ont animé les enquêtes centrales du CRH pendant ces années. Comme le départ d'E. Le Roy Ladurie à l'université Paris VII réduisait visiblement le poids des recherches sur le recrutement militaire au XIXe siècle, avec son successeur F. Furet le programme labroussien d'histoire sociale des origines de la France bourgeoise passa au centre des activités du CRH. Le projet primitif d'une « sociologie historique des origines de la France bourgeoise » regroupait à lui seul sept enquêtes flanquées par celles sur les cahiers de doléances de 1789 et sur la correspondance de Rousseau. La problématique des enquêtes sur les structures sociales de la France bourgeoise s'est lentement infléchie vers l'analyse des structures urbaines. D'abord concentré primordialement sur Paris, ces enquêtes ouvraient des perspectives nouvelles pour une histoire sociale de la France industrielle contemporaine ${ }^{14}$. 
17 Les recherches sur la civilisation matérielle (la consommation, l'atlas mondial, les enquêtes du groupe d'archéologie médiévale ${ }^{15}$ poursuivaient des chemins tracés dans la période braudelienne, mais, sauf exceptions rares en marge des activités du Centre ${ }^{16}$, la problématique braudelienne fut abandonnée. A l'intersection des traditions de recherche de Braudel et de Labrousse se placent les enquêtes sur la production agricole animées par Le Roy Ladurie et J. Goy où le CRH ne faisait que coordonner des recherches extérieures.

Comme aux origines du $\mathrm{CRH}$, période des recherches sur la Méditerranée braudelienne, la démarche scientifique sous-jacente à toutes ces enquêtes est commandée par une stratégie conservatrice d'innovation (C. Knorr) caractérisée par l'application d'une solution déjà confirmée à des matériaux encore inexplorés. Les enquêtes sur les dîmes suivent-hormis de rares exceptions-le programme et la problématique exemplairement mis en oeuvre au début des années 1960, notamment par Le Roy Ladurie lui-même. Les projets de sociologie historique des origines de la France bourgeoise ou de démographie historique (Châtillonais, Catasto florentin, Vexin) relèvent de la même logique.

Tableau 12. Enquêtes collectives du CRH 1968-1974

\begin{tabular}{|c|c|}
\hline \multicolumn{2}{|c|}{ Sociologie historique des origines de la France bourgeoise (1969.76) } \\
\hline $\begin{array}{l}\text { 1. Sociologie des armées de la Révolution Fran- } \\
\text { caise } 1968.1971 \text { ) }\end{array}$ & J.P. Bertaud (dir.) \\
\hline $\begin{array}{l}\text { 2. Les notables du Grand Empire (1969-1976, Pre- } \\
\text { rnière phase : dépouillement des listes electorales } \\
\text { et traitement par ordinateur des données collectées } \\
\text { sur les notables }(-1976) \text { Deuxièrne phase : publica. } \\
\text { tion des biographies sociales des notables dépar- } \\
\text { tementaux (1976-) }\end{array}$ & $\begin{array}{l}\text { Bergeron, Forster, Chaussi- } \\
\text { nand, Nogaret (dir.) }\end{array}$ \\
\hline $\begin{array}{l}\text { 3. Sociologie urbaine du XIXe siècle parisien } \\
(1969-1973)\end{array}$ & Daumard (dir) \\
\hline $\begin{array}{l}\text { 4. Les mécanismes de la croissance urbaine a Paris } \\
\text { au XIXes. }\end{array}$ & $\begin{array}{l}\text { Roncayolo, Bergeron (dir.), } \\
\text { Ceau, Mallet, Chatelain }\end{array}$ \\
\hline $\begin{array}{l}\text { 5. L.anoblissement au XVIIIle s. et la Compagnie } \\
\text { des Sécretaires du Roi (1972-1976): dépouillement } \\
\text { de la série V2 des Archives Nationales (dossiers de } \\
\text { réception des Sécretaires du Roi 1672-1789) }\end{array}$ & $\begin{array}{l}\text { Bien, Chaussinand, Furet, } \\
\text { Meyer. }\end{array}$ \\
\hline \multicolumn{2}{|c|}{ Cahiers de doléances de l'ancienne France (1969-1975) } \\
\hline 1. Les cahiers de 1614 & Richet (dir.) \\
\hline 2. Les cahiers de 1789 & Furet (dir.) \\
\hline \multicolumn{2}{|c|}{ 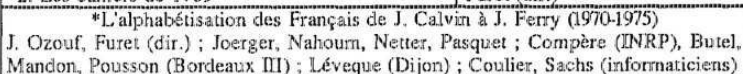 } \\
\hline \multirow{2}{*}{\multicolumn{2}{|c|}{$\begin{array}{l}\text { *Le catasto florentin de } 1427 \text { (1968-1974) } \\
\begin{array}{l}\text { Klapisch, Herlihy (Unniv. du Wisconsin) (dir.); Melis, Conti (Italie); Triantaphylli- } \\
\text { dou: Asdrachas (CNRS) }\end{array} \\
\text { "Dépouillernent mécanographique et analyse de contenu de la correspondance de } \\
\text { Rousseau (1968-1974) } \\
\text { Furet Roche (ENS StCloud): Launay (Nice) }\end{array}$}} \\
\hline & \\
\hline \multicolumn{2}{|c|}{$\begin{array}{l}\text { Enquête rationale sur la consormmation (1971-1973) } \\
\text { Coordination : Goy (CRH), Bernassar (Toulouse) }\end{array}$} \\
\hline $\begin{array}{l}\text { Consommation, notamment alimentaire, en Eu- } \\
\text { rope et en Afrique du Nord XIVe-XXes. }\end{array}$ & $\begin{array}{l}\text { Une quarantaine de chercheurs } \\
\text { dont } 15 \text { du CRH }\end{array}$ \\
\hline \multicolumn{2}{|c|}{$\begin{array}{l}\text { La littérature des exempla au Moyen âge } \\
\text { Le Goff (dir.), Brémond, Schmitt (1973-1986 et sv) }\end{array}$} \\
\hline $\begin{array}{l}\text { Enquête internationale sur les revenus décin } \\
\text { du XIVe au XIXes (1973 } \\
\text { Goy, Le Roy Ladurie (dir.). Coordination des équi } \\
\text { colloque intemational à Paris en } 1977 \text { et du } 7 \text { e Cor } \\
\text { nale d'histoire économique en } 1978\end{array}$ & $\begin{array}{l}\text { maux et la rente foncière } \\
\text {-1977) } \\
\text { ipes nationales et préparation du } \\
\text { ngrès de l'Association internatio- }\end{array}$ \\
\hline
\end{tabular}

Les enquêtes précédées d'un astérisque ont utilisé un ordinateur. ${ }^{17}$

19 Le cas des enquêtes animées par F. Furet, J. Ozouf, D. Richet est plus complexe. Partis d'une problématique labroussienne et engagés dans le combat d'une histoire quantitative conquérant les terrains hostiles de l'histoire intellectuelle ou culturelle dans les années 1960, ces groupes infléchirent peu à peu leur problématique. Le monopole des séries quantifiables en tant que sources primaires cédait le pas à une approche documentaire beaucoup plus ouverte qui allait de pair avec une remise en question des modèles à étages communs aux traditions annalistes et marxistes de l'historiographie française. Langage politique, réseaux intellectuels, histoire de l'enseignement et de l'alphabétisation comme 
aspects fondamentaux de la transformation sociale du $\mathrm{XVI}^{\mathrm{e}}$ au $\mathrm{XIX}^{\mathrm{e}}$ siècle, de nouvelles techniques documentaires et une modélisation théorique hétérodoxe permirent de revenir sur ces sujets « classiques » et en proposer une interprétation « révisionnisme ». Ce type d'innovation par « hérésie » fonctionna comme catalyseur pour toute une série de recherches qui cherchaient à explorer le champ à contours encore flous d'une histoire sociale des phénomènes culturels et politiques. Le succès de ces enquêtes finit par ronger les concepts de base qui avaient cimenté les travaux des disciples de Braudel et de Labrousse pendant plus de 20 ans, éléments d'une pensée collective routinière, tel le primat des structures économiques, démographiques ou géographiques, telle l'explication du politique par les structures et les conjonctures socio-économiques, telle la dichotomie entre événements et structures.

20 Au milieu des années 1970, le Centre de recherches historiques pouvait gérer ses affaires en s'appuyant largement sur son prestige intellectuel accumulé et sur sa taille, deux facteurs qui lui donnaient une position centrale sur le marché à la fois bureaucratique et politique des subventions publiques à la recherche. Le rayonnement intellectuel - surtout à l'étranger - se poursuivait dans une période marquée par un retournement brusque de la conjoncture d'expansion. Dans cette situation, la logique de conservation des acquis en termes de ressources financières, de postes disponibles et de personnel déjà embauché, s'est imposée. Une telle stratégie - en vérité plutôt le résultat d'une série de compromis entre intérêts divergents et gestion réaliste des succès scientifiques - interdisait toute rupture avec le passé et toute réorientation des activités de recherche. Les grandes enquêtes d'histoire quantitative prirent fin en 1974 sans que le CRH en relançât d'autres de même envergure. Une minorité de chercheurs tentait de sortir des pistes balisées pour ouvrir de nouveaux chantiers, comme l'enquête sur l'histoire des universités ou sur les archives orales de la France. Bien que les propositions n'aient pas manqué - ouverture sur l'histoire contemporaine et sur l'histoire comparative - le poids du passé, sous forme de structures objectives et d'héritage intellectuel à gérer et à défendre, avait tendance à l'emporter. "En effet, le Centre est essentiellement un héritier », cette conclusion du directeur du CRH, F. Furet, en 1972, résumait bien les problèmes d'une nouvelle période $\mathrm{du} \mathrm{CRH}^{18}$.

21 L'analyse des activités du CRH dans cette dernière phase d'expansion serait incomplète si l'on ne considérait pas ses publications. L'héritage des années 1950 - une politique d'édition offensive - a été poursuivi avec une certaine modération tout au long des années 1960, à la différence que les collections du CRH reflétaient plus directement ses activités de recherche même. L'augmentation des postes permettait un nombre croissant d'enquêtes dont les résultats alimentèrent des collections toujours plus diversifiées. Aux quatre séries des années 1950, s'ajoutèrent la collection d'histoire rurale « Les hommes et la terre", celle de "Documents et recherches sur l'économie des pays byzantins", résultat de l'intégration temporaire du centre de recherche de Lemerle dans le CRH, et la collection « Démographie et société ». 
Tableau 13. Nombre de volumes publiés dans les collections du CRH. I. 1963-1974

\begin{tabular}{|c|c|c|c|c|c|c|c|}
\hline Collections Années & $\begin{array}{l}1.951 \\
1.962\end{array}$ & $\begin{array}{l}1953 \\
1954\end{array}$ & $\begin{array}{l}1965 \\
1966\end{array}$ & $\begin{array}{l}1967 \\
1958\end{array}$ & $\begin{array}{l}1969 \\
1970\end{array}$ & $\begin{array}{l}1971 \\
1972\end{array}$ & $\begin{array}{l}1973 \\
1974\end{array}$ \\
\hline Affalres et pens d'alfiures & 28 & 1 & 2 & 2 & 3 & - & 4 \\
\hline Ports-Routes-T'rafics & 24 & 6 & 3 & 5 & 4 & $=$ & $\therefore$ \\
\hline Prix-Monnaie Conjoncture & 6 & $\therefore$ & 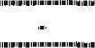 & 1 & 2 & 1 & 1 \\
\hline Les hormenes el lat terre & 7 & 3 & 4 & 3 & $\therefore$ & 1 & $\therefore$ \\
\hline Doc et rech byzanitins & 5 & 2 & 1 & $\therefore$ & 1 & 2 & 1 \\
\hline Dénog grephie et sociéte & 7 & 1 & 1 & $\therefore$ & 5 & 1 & 1 \\
\hline L ivree soct societé & 1 & - & 1 & - & 2 & - & $\therefore$ \\
\hline Mencires de photo-interpr. & -... & 11 & 2 & 2 & 2 & 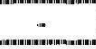 & $\therefore$ \\
\hline Industrie et artisantat & - na.. & . & 2 & 4 & 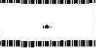 & 1 & 2 \\
\hline Cahilers des études nurales & ... & 1 & - & n... & . & 1 & $\therefore-$ \\
\hline Civilisations et soofetes & (n).... & 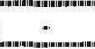 & 3 & 10 & 6 & 1] & 1.1. \\
\hline Le savoir historigue & -... & $\therefore$ & $\therefore$ & $=$ & $=$ & 3 & 5 \\
\hline Archéologie el civilisalion & -... & 1 & 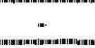 & 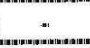 & $\therefore$ & $\therefore$ & 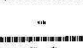 \\
\hline Wonnibre de volu wers & 78 & 1.6 & 19 & 27 & 25 & 21 & 22 \\
\hline Wombre de collections & 7 & $B$ & 9 & 7 & 8 & 8 & 7 \\
\hline
\end{tabular}

Tableau 13. Nombre de volumes publiés dans les collections du CRH. II. 1975-1980

\begin{tabular}{|c|c|c|c|c|}
\hline $\begin{array}{l}\text { Collections Années } \\
\end{array}$ & $\begin{array}{l}1963 \\
1974\end{array}$ & $\begin{array}{l}1975 \\
1976\end{array}$ & $\begin{array}{l}1977 \\
1978\end{array}$ & $\begin{array}{l}1979 \\
1980\end{array}$ \\
\hline Affarres et gens d'affacires & 9 & - & 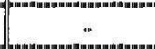 & - \\
\hline Ports-Routes-Tiralfios & 18 & - & (- & 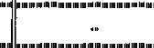 \\
\hline Prix - Montraie -Conjonctura & 5 & - & - & - \\
\hline Lews hormones of lat herre & 11 & 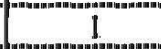 & - & il \\
\hline Doc. en rech. byzantins & 7 & - & - & tis \\
\hline Dénographie el société & 9 & - & - & - \\
\hline Live et societé & 3 & $=$ & (n) & $=$ \\
\hline Mémoires de photo-ninterpr. & 7 & - & - & . \\
\hline Inolustrie ets artisanat & 9 & (- & - & 1 \\
\hline Cahiers des éndes rurales & 2 & 1 & . & 1 \\
\hline Civilisations el societes & 41 & 110 & 1.4 & 11 \\
\hline Le savoir historigue & 8 & 3 & 2 & - \\
\hline Archeologie en civilisation. & 1 & 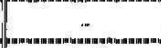 & $\ldots$ & - \\
\hline Nombre de volurnes & 130 & 15 & 15 & 13 \\
\hline Nornbre ols colledtions & 10 & 4 & 2 & 5 \\
\hline
\end{tabular}

Les tableaux 13 montrent que cette deuxième phase d'expansion s'est prolongée jusqu'aux années 1969-1970, point culminant des activités d'édition du CRH suivie d'une période de stagnation à un niveau encore élevé jusqu'en 1973-1974, qui marque une rupture à cet égard : avec la fin des quatre collections classiques du Centre ("Affaires et gens d'affaires »; «Ports - Routes - Trafics »; «Prix - Monnaie - Conjoncture »; " Démographie et société ») c'est tout une série de recherches centrées sur l'histoire des débuts du capitalisme moderne et sur l'histoire économique et sociale de l'Ancien Régime qui s'achève. Point d'attraction nouveau depuis la fin des années 1960, la collection "Civilisations et sociétés" reflète la nouvelle orientation des recherches sous les rubriques nouvelles d'anthropologie historique ou d'histoire des mentalités. En même temps, les collections du CRH cessèrent de publier les principaux résultats de ses membres. À l'origine de cette marginalisation relative de ce secteur d'activités du Centre, 
on retrouve les changements intervenus dans tout le champ de l'édition scientifique avec l'élargissement du public étudiant et du marché des biens culturels en général. Les éditions du CRH s'adressaient à un public restreint et dans la perspective d'une longue durée de distribution : ces deux aspects les marginalisèrent sur un marché où paraissaient les premières éditions allégées de thèses en format de poche et la concurrence directe des grandes maisons d'édition attirées par la renommée croissante des auteurs du CRH ou de la $\mathrm{VI}^{\mathrm{e}}$ Section. L'augmentation des prix de production à partir de 1972-1973 pouvait en plus inciter les nouvelles directions de l'École et du CRH à réviser largement à la baisse leurs ambitions en matière de publication scientifique. Les positions privilégiées que des membres du CRH (Le Goff, Goy, Furet, Richet) ont pu établir dans le monde de l'édition en tant que directeurs de collections et la participation étroite d'autres membres du Centre aux réseaux de production culturelle offraient des perspectives qui, à court terme, dépassaient largement les ressources des collections scientifiques de la maison. Si l'on compare les titres des collections du CRH aux listes des publications de ses membres dans les rapports d'activités, on peut prendre la mesure de la marginalisation de cette activité du Centre en fin de période d'expansion. Des 50 livres français mentionnés dans la liste des publications des membres du CRH en 1978 et 1979, 5 titres seulement ont paru dans les collections de l'EHESS ${ }^{19}$.

\section{NOTES}

1. A. Daumard, «Paris et les archives de l'enregistrement », Annales ESC, 1958, p. 289-303.

2. Sur la position institutionnelle de Labrousse, voir les indicateurs recueillis dans P. Bourdieu, Homo academicus, op. cit., p. 122 et 124.

3. Ch. Charle, "Entretien avec E. Labrousse ", Actes de la Recherche en Sciences Sociales, $\mathrm{n}^{\circ}$ 32-33, 1980, p. 111-127.

4. CRH, Rapport d'activité 1968-69, p. 1, archives EHESS, fonds Velay.

5. Cf. les articles programmatiques d'E. Le Roy Ladurie, «L'historien et l'ordinateur » (1968) et «Du quantitatif en histoire la VIe section de l'École pratique des Hautes Études » (1967), Le territoire de l'historien, Paris, 1973, p. 11-22 et 23-37, et celui de F. Furet, « L'histoire quantitative et la construction du fait historique ", Annales ESC, 1971, p. 63-75.

6. F. Furet, «L'histoire quantitative... » art. cit. p. 67.

7. Le Roy Ladurie, demande de vacations supplémentaires au CNRS, 1966, p. 4, archives EHESS, fonds Velay.

8. D. Roche, «De l'histoire sociale à l'histoire socioculturelle ", Mélanges de l'École française de Rome, XCI (1979), p. 7-19.

9. Cf. les tentatives de définition d'André Burguière, "L'anthropologie historique ", La Nouvelle histoire. Paris, 1978, p. 37-61.

10. Cf., p. ex., la conférence d'E. Le Roy Ladurie à Toronto en décembre 1967, « Du quantitatif en histoire ", op. cit.

11. Les Annales ESC des années 1969-1972 se firent l'écho fidèle des enquêtes en cours.

12. Cetina-Knorr, Die Fabrikation von Erkenntnis, op. cit., p. 64.

13. CRH, Rapport d'activités 1969-70, 1971-1972, 1972-1974, archives EHESS. 
14. Sur les changements de perspectives qu'une telle évolution impliquait, cf. L. Bergeron, M. Roncayolo, «De la ville préindustrielle à la ville industrielle. Essai sur l'historiographie française ", Quaderni storici, 1974, p. 827-876.

15. La liste devient plus longue si l'on ajoute des enquêtes plus personnelles menées au CRH, comme les recherches sur les campagnes de Meaux de M. Baulant ou sur les salaires au XvI siècles de $\mathrm{M}$. Baulant et $\mathrm{C}$. Beutler.

16. Comme, p. ex., l'enquête de A. et B. Tenenti sur l'assurance maritime à Venise et à Raguse (1969-1980).

17. Bergeron, Chaussinand, Nogaret, Les masses de granit. Cent mille notables du Grand Empire, 1979. Furet, J. Ozouf, Lire et Écrire, 2 vol., 1977, Klapisch, Herlihy, Les Toscans et leurs familles. Une étude du catasto florentin de 1427, 1977. Roche, "Les primitifs du Rousseauisme ", Annales ESC, 1971, p. 151-172 ; Launay, Le vocabulaire politique dans les ceuvres de Rousseau, thèse complémentaire, 1970. Pour l'enquête nationale sur la consommation, cf Actes du $\mathrm{II}^{\mathrm{e}}$ Congrès national des historiens économistes (1973), 1975. Brémond, Le Goff, Schmitt, L'exemplum, Turnhout, 1982.

18. Procès-verbal de la réunion de chercheurs du CRH du 11 décembre 1972, archives EHESS, fonds Velay.

19. CRH, Rapport d'activités 1978-1980, Paris. s.d. 